The LIGO Collaboration

This article has been downloaded from IOPscience. Please scroll down to see the full text article.

2010 Class. Quantum Grav. 27069802

(http://iopscience.iop.org/0264-9381/27/8/089602)

View the table of contents for this issue, or go to the journal homepage for more

Download details:

IP Address: 18.7.29.240

The article was downloaded on 28/05/2011 at 13:15

Please note that terms and conditions apply. 


\section{The LIGO Collaboration}

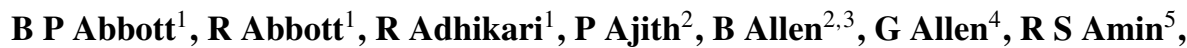

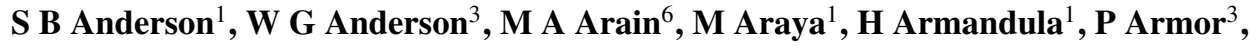

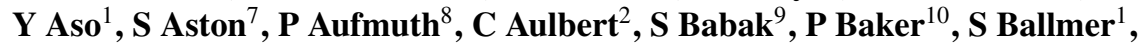
C Barker ${ }^{11}$, D Barker ${ }^{11}$, B Barr ${ }^{12}$, P Barriga ${ }^{13}$, L Barsotti ${ }^{14}$, M A Barton ${ }^{1}$, I Bartos ${ }^{15}$, R Bassiri ${ }^{12}$, M Bastarrika ${ }^{12}$, B Behnke ${ }^{9}$, M Benacquista ${ }^{16}$, J Betzwieser ${ }^{1}$, P T Beyersdorf ${ }^{17}$, I A Bilenko ${ }^{18}$, G Billingsley ${ }^{1}$, R Biswas ${ }^{3}$, E Black ${ }^{1}$, J K Blackburn ${ }^{1}$, L Blackburn $^{14}$, D Blair ${ }^{13}$, B Bland ${ }^{11}$, T P Bodiya ${ }^{14}$, L Bogue ${ }^{19}$, R Bork ${ }^{1}$, V Boschi ${ }^{1}$, S Bose ${ }^{20}$, P R Brady ${ }^{3}$, V B Braginsky ${ }^{18}$, J E Brau ${ }^{21}$, D O Bridges ${ }^{19}$, M Brinkmann ${ }^{2}$, A F Brooks ${ }^{1}$, D A Brown ${ }^{22}$, A Brummit ${ }^{23}$, G Brunet ${ }^{14}$, A Bullington ${ }^{4}$, A Buonanno ${ }^{24}$, O Burmeister ${ }^{2}$, R L Byer ${ }^{4}$, L Cadonati ${ }^{25}$, J B Camp ${ }^{26}$, J Cannizzo ${ }^{26}$, K C Cannon ${ }^{1}$, J Cao ${ }^{14}$, L Cardenas ${ }^{1}$, S Caride ${ }^{27}$, G Castaldi ${ }^{28}$, S Caudill ${ }^{5}, \mathbf{M}$ Cavaglià $^{29}$, C Cepeda ${ }^{1}$, T Chalermsongsak ${ }^{1}$, E Chalkley ${ }^{12}$, P Charlton $^{30}$, S Chatterji ${ }^{1}$, S Chelkowski $^{7}$, Y Chen ${ }^{9,31}$, N Christensen ${ }^{32}$, C T Y Chung ${ }^{33}$, D Clark ${ }^{4}$, J Clark ${ }^{34}$, J H Clayton ${ }^{3}$, T Cokelaer $^{34}$, C N Colacino ${ }^{35}$, R Conte ${ }^{36}$, D Cook ${ }^{11}$, T R C Corbitt ${ }^{14}$, N Cornish ${ }^{10}$, D Coward $^{13}$, D C Coyne ${ }^{1}$, J D E Creighton ${ }^{3}$, T D Creighton ${ }^{16}$, A M Cruise ${ }^{7}$, R M Culter ${ }^{7}$, A Cumming ${ }^{12}$, L Cunningham ${ }^{12}$, S L Danilishin ${ }^{18}$, K Danzmann ${ }^{2,8}$, B Daudert ${ }^{1}$, G Davies ${ }^{34}$, E J Daw ${ }^{37}$, D DeBra ${ }^{4}$, J Degallaix ${ }^{2}$, V Dergachev ${ }^{27}$, S Desai ${ }^{38}$, R DeSalvo ${ }^{1}, \mathbf{S}$ Dhurandhar ${ }^{39}, \mathbf{M}^{\text {Díaz }}{ }^{16}$, A Dietz ${ }^{34}$, F Donovan ${ }^{14}$, K L Dooley ${ }^{6}$, E E Doomes ${ }^{40}$, R W P Drever ${ }^{41}$, J Dueck ${ }^{2}$, I Duke ${ }^{14}$, J-C Dumas ${ }^{13}$, J G Dwyer ${ }^{15}$,

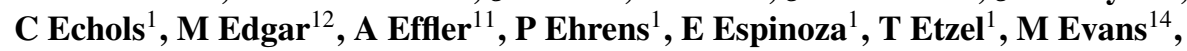

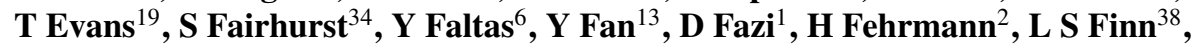

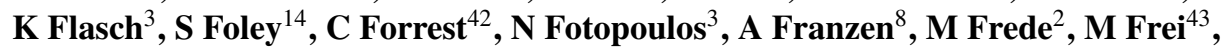

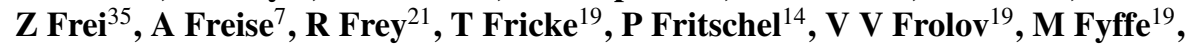
V Galdi $^{28}$, J A Garofoli ${ }^{22}$, I Gholami ${ }^{9}$, J A Giaime ${ }^{5,19}$, S Giampanis ${ }^{2}$, K D Giardina ${ }^{19}$, K Goda ${ }^{14}$, E Goetz ${ }^{27}$, L M Goggin ${ }^{3}$, G González ${ }^{5}$, M L Gorodetsky ${ }^{18}$, S Goßler ${ }^{2}$,

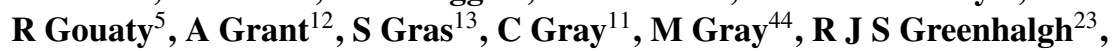
A M Gretarsson ${ }^{45}$, F Grimaldi ${ }^{14}$, R Grosso ${ }^{16}$, H Grote $^{2}$, S Grunewald $^{9}$, M Guenther ${ }^{11}$,

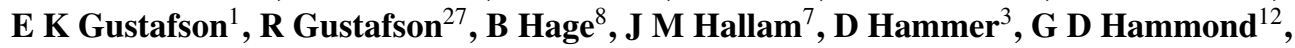
C Hanna ${ }^{1}$, J Hanson ${ }^{19}$, J Harms ${ }^{46}$, G M Harry ${ }^{14}$, I W Harry ${ }^{34}$, E D Harstad ${ }^{21}$, K Haughian $^{12}$, K Hayama ${ }^{16}$, J Heefner ${ }^{1}$, I S Heng ${ }^{12}$, A Heptonstall ${ }^{1}$, M Hewitson ${ }^{2}$,

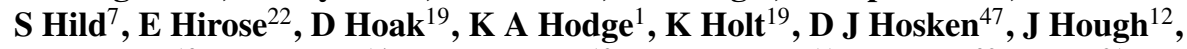

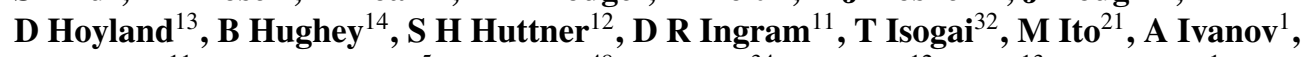
B Johnson ${ }^{11}$, W W Johnson ${ }^{5}$, D I Jones ${ }^{48}$, G Jones ${ }^{34}$, R Jones ${ }^{12}$, L Ju ${ }^{13}$, P Kalmus ${ }^{1}$, V Kalogera ${ }^{49}$, S Kandhasamy ${ }^{46}$, J Kanner ${ }^{24}$, D Kasprzyk ${ }^{7}$, E Katsavounidis ${ }^{14}$, K Kawabe $^{11}$, S Kawamura ${ }^{50}$, F Kawazoe ${ }^{2}$, W Kells ${ }^{1}$, D G Keppel ${ }^{1}$, A Khalaidovski ${ }^{2}$, F Y Khalili ${ }^{18}$, R Khan ${ }^{15}$, E Khazanov ${ }^{51}$, P King ${ }^{1}$, J S Kissel ${ }^{5}$, S Klimenko ${ }^{6}$, K Kokeyama $^{50}$, V Kondrashov ${ }^{1}$, R Kopparapu ${ }^{38}$, S Koranda ${ }^{3}$, D Kozak ${ }^{1}$, B Krishnan ${ }^{9}$, R Kumar ${ }^{12}$, P Kwee ${ }^{8}$, P K Lam ${ }^{44}$, M Landry ${ }^{11}$, B Lantz ${ }^{4}$, A Lazzarini ${ }^{1}$, H Lei ${ }^{16}$, M Lei ${ }^{1}$, N Leindecker ${ }^{4}$, I Leonor ${ }^{21}, \mathrm{C} \mathrm{Li}^{31}, \mathbf{H}$ Lin $^{6}, \mathbf{P}$ E Lindquist ${ }^{1}$, T B Littenberg ${ }^{10}$, N A Lockerbie ${ }^{52}$, D Lodhia ${ }^{7}, \mathbf{M}$ Longo $^{28}, \mathbf{M ~ L o r m a n d ~}^{19}, \mathbf{P}$ Lu $^{4}, \mathbf{M}$ Lubinski $^{11}$, A Lucianetti ${ }^{6}$, H Lück ${ }^{2,8}$, B Machenschalk ${ }^{9}$, M MacInnis ${ }^{14}$, M Mageswaran ${ }^{1}$, 
K Mailand ${ }^{1}$, I Mandel ${ }^{49}$, V Mandic ${ }^{46}$, S Márka ${ }^{15}$, Z Márka ${ }^{15}$, A Markosyan ${ }^{4}$, J Markowitz ${ }^{14}$, E Maros ${ }^{1}$, I W Martin ${ }^{12}$, R M Martin ${ }^{6}$, J N. Marx ${ }^{1}$, K Mason ${ }^{14}$, F Matichard $^{5}$, L Matone ${ }^{15}$, R A Matzner ${ }^{43}$, N Mavalvala ${ }^{14}$, R McCarthy ${ }^{11}$, D E McClelland ${ }^{44}$, S C McGuire ${ }^{40}$, M McHugh ${ }^{53}$, G McIntyre ${ }^{1}$, D J A McKechan ${ }^{34}$, K McKenzie $^{44}$, M Mehmet ${ }^{2}$, A Melatos ${ }^{33}$, A C Melissinos ${ }^{42}$, D F Menéndez ${ }^{38}$, G Mendell ${ }^{11}$, R A Mercer ${ }^{3}$, S Meshkov ${ }^{1}$, C Messenger ${ }^{2}$, M S Meyer ${ }^{19}$, J Miller ${ }^{12}$, J Minelli $^{38}$, Y Mino ${ }^{31}$, V P Mitrofanov ${ }^{18}$, G Mitselmakher ${ }^{6}$, $\mathbf{R}$ Mittleman $^{14}$, O Miyakawa $^{1}$, B Moe ${ }^{3}$, S D Mohanty ${ }^{16}$, S R P Mohapatra ${ }^{25}$, G Moreno ${ }^{11}$, T Morioka ${ }^{50}$, K Mors $^{2}$, K Mossavi ${ }^{2}$, C MowLowry ${ }^{44}$, G Mueller ${ }^{6}$, H Müller-Ebhardt ${ }^{2}$, D Muhammad $^{19}$, S Mukherjee ${ }^{16}$, H Mukhopadhyay ${ }^{39}$, A Mullavey ${ }^{44}$, J Munch $^{47}$,

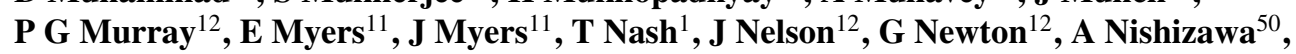
K Numata ${ }^{26}$, J O'Dell ${ }^{23}$, B O'Reilly ${ }^{19}$, R O'Shaughnessy ${ }^{38}$, E Ochsner $^{24}$, G H Ogin ${ }^{1}$, D J Ottaway ${ }^{47}$, R S Ottens ${ }^{6}$, H Overmier ${ }^{19}$, B J Owen ${ }^{38}$, Y Pan ${ }^{24}$, C Pankow ${ }^{6}$,

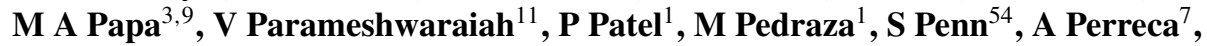
V Pierro ${ }^{28}$, I M Pinto ${ }^{28}$, M Pitkin ${ }^{12}$, H J Pletsch ${ }^{2}$, M V Plissi ${ }^{12}$, F Postiglione $^{36}$, M Principe ${ }^{28}$, R Prix ${ }^{2}$, L Prokhorov ${ }^{18}$, O Puncken ${ }^{2}$, V Quetschke $^{6}$, F J Raab ${ }^{11}$, D S Rabeling ${ }^{44}$, H Radkins ${ }^{11}$, P Raffai ${ }^{35}$, Z Raics ${ }^{15}$, N Rainer ${ }^{2}$, M Rakhmanov ${ }^{16}$, V Raymond ${ }^{49}$, C M Reed ${ }^{11}$, T Reed ${ }^{55}$, H Rehbein ${ }^{2}$, S Reid ${ }^{12}$, D H Reitze ${ }^{6}$, R Riesen ${ }^{19}$, K Riles $^{27}$, B Rivera ${ }^{11}$, P Roberts ${ }^{56}$, N A Robertson ${ }^{1,12}$, C Robinson ${ }^{34}$, E L Robinson ${ }^{9}$, S Roddy ${ }^{19}$, C Röver ${ }^{2}$, J Rollins ${ }^{15}$, J D Romano ${ }^{16}$, J H Romie ${ }^{19}$, S Rowan ${ }^{12}$, A Rüdiger ${ }^{2}$, P Russell $^{1}$, K Ryan ${ }^{11}$, S Sakata ${ }^{50}$, L Sancho de la Jordana ${ }^{57}$, V Sandberg ${ }^{11}$,

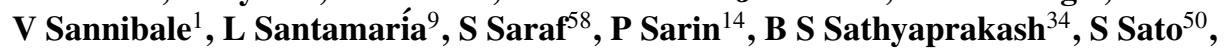
M Satterthwaite ${ }^{44}, \mathbf{P}$ R Saulson ${ }^{22}, \mathbf{R}_{\text {Savage }^{11}}$, P Savov $^{31}$, M Scanlan $^{55}$, R Schilling $^{2}$, R Schnabel $^{2}$, R Schofield ${ }^{21}$, B Schulz ${ }^{2}$, B F Schutz ${ }^{9,34}$, P Schwinberg ${ }^{11}$, J Scott ${ }^{12}$, S M Scott ${ }^{44}$, A C Searle ${ }^{1}$, B Sears ${ }^{1}$, F Seifert ${ }^{2}$, D Sellers ${ }^{19}$, A S Sengupta ${ }^{1}$, A Sergeev ${ }^{51}$, B Shapiro ${ }^{14}$, P Shawhan ${ }^{24}$, D H Shoemaker ${ }^{14}$, A Sibley ${ }^{19}$, X Siemens ${ }^{3}$, D Sigg ${ }^{11}$, S Sinha ${ }^{4}$, A M Sintes ${ }^{57}$, B J J Slagmolen ${ }^{44}$, J Slutsky ${ }^{5}$, J R Smith ${ }^{22}$, M R Smith ${ }^{1}$, N D Smith ${ }^{14}$, K Somiya ${ }^{31}$, B Sorazu ${ }^{12}$, A Stein ${ }^{14}$, L C Stein ${ }^{14}$, S Steplewski ${ }^{20}$, A Stochino ${ }^{1}$, R Stone ${ }^{16}$, K A Strain ${ }^{12}, \mathbf{S ~ S t r i g i n}{ }^{18}, \mathbf{A}$ Stroeer $^{26}$, A L Stuver ${ }^{19}$, T Z Summerscales ${ }^{56}$, K-X Sun ${ }^{4}$, M Sung ${ }^{5}$, P J Sutton ${ }^{34}$, G P Szokoly ${ }^{35}$, D Talukder ${ }^{20}$, L Tang ${ }^{16}$, D B Tanner ${ }^{6}$, S P Tarabrin ${ }^{18}$, J R Taylor ${ }^{2}$, R Taylor ${ }^{1}$, J Thacker ${ }^{19}$, K A Thorne ${ }^{19}$, K S Thorne ${ }^{31}$, A Thüring ${ }^{8}$, K V Tokmakov ${ }^{12}$, C Torres ${ }^{19}$, C Torrie ${ }^{1}$, G Traylor $^{19}$, M Trias ${ }^{57}$, D Ugolini ${ }^{59}$, J Ulmen $^{4}$, K Urbanek $^{4}$, H Vahlbruch ${ }^{8}$, M Vallisneri ${ }^{31}$, C Van Den Broeck ${ }^{34}, \mathbf{M}$ V van der Sluys ${ }^{49}$, A A van Veggel $^{12}$, S Vass ${ }^{1}$, R Vaulin ${ }^{3}$, A Vecchio ${ }^{7}$, J Veitch $^{7}, \mathbf{P}$ Veitch $^{47}$, C Veltkamp ${ }^{2}$, A Villar ${ }^{1}$, C Vorvick ${ }^{11}$, S P Vyachanin ${ }^{18}$, S J Waldman ${ }^{14}$, L Wallace ${ }^{1}$, R L Ward ${ }^{1}$, A Weidner ${ }^{2}$, M Weinert ${ }^{2}$,

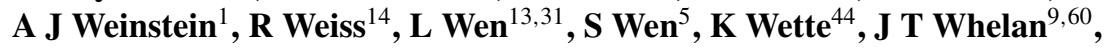
S E Whitcomb ${ }^{1}$, B F Whiting ${ }^{6}$, C Wilkinson ${ }^{11}$, P A Willems ${ }^{1}$, H R Williams ${ }^{38}$, L Williams $^{6}$, B Willke ${ }^{2,8}$, I Wilmut ${ }^{23}$, L Winkelmann ${ }^{2}$, W Winkler ${ }^{2}$, C C Wipf $^{14}$, A G Wiseman ${ }^{3}$, G Woan ${ }^{12}$, R Wooley ${ }^{19}$, J Worden ${ }^{11}$, W Wu ${ }^{6}$, I Yakushin ${ }^{19}$, H Yamamoto ${ }^{1}$, Z Yan $^{13}$, S Yoshida ${ }^{61}$, M Zanolin ${ }^{45}$, J Zhang ${ }^{26}$, L Zhang ${ }^{1}$, C Zhao ${ }^{13}$, N Zotov $^{55}$, M E Zucker ${ }^{14}$, H zur Mühlen ${ }^{8}$, and J Zweizig ${ }^{1}$ (The LIGO Scientific Collaboration, http://www.ligo.org)

${ }^{1}$ LIGO - California Institute of Technology, Pasadena, CA 91125, USA

${ }^{2}$ Albert-Einstein-Institut, Max-Planck-Institut für Gravitationsphysik, D-30167 Hannover, Germany

${ }^{3}$ University of Wisconsin-Milwaukee, Milwaukee, WI 53201, USA

${ }^{4}$ Stanford University, Stanford, CA 94305, USA 
${ }^{5}$ Louisiana State University, Baton Rouge, LA 70803, USA

${ }^{6}$ University of Florida, Gainesville, FL 32611, USA

${ }^{7}$ University of Birmingham, Birmingham, B15 2TT, United Kingdom

${ }^{8}$ Leibniz Universität Hannover, D-30167 Hannover, Germany

${ }^{9}$ Albert-Einstein-Institut, Max-Planck-Institut für Gravitationsphysik, D-14476 Golm, Germany

${ }^{10}$ Montana State University, Bozeman, MT 59717, USA

${ }^{11}$ LIGO - Hanford Observatory, Richland, WA 99352, USA

${ }^{12}$ University of Glasgow, Glasgow, G12 8QQ, United Kingdom

${ }^{13}$ University of Western Australia, Crawley, WA 6009, Australia

${ }^{14}$ LIGO - Massachusetts Institute of Technology, Cambridge, MA 02139, USA

${ }^{15}$ Columbia University, New York, NY 10027, USA

${ }^{16}$ The University of Texas at Brownsville and Texas Southmost College, Brownsville, TX 78520, USA

${ }^{17}$ San Jose State University, San Jose, CA 95192, USA

${ }^{18}$ Moscow State University, Moscow, 119992, Russia

${ }^{19}$ LIGO - Livingston Observatory, Livingston, LA 70754, USA

${ }^{20}$ Washington State University, Pullman, WA 99164, USA

${ }^{21}$ University of Oregon, Eugene, OR 97403, USA

${ }^{22}$ Syracuse University, Syracuse, NY 13244, USA

${ }^{23}$ Rutherford Appleton Laboratory, HSIC, Chilton, Didcot, Oxon OX11 0QX, United Kingdom

${ }^{24}$ University of Maryland, College Park, MD 20742 USA

${ }^{25}$ University of Massachusetts - Amherst, MA 01003 USA

${ }^{26}$ NASA/Goddard Space Flight Center, Greenbelt, MD 20771, USA

${ }^{27}$ University of Michigan, Ann Arbor, MI 48109, USA

${ }^{28}$ University of Sannio at Benevento, I-82100 Benevento, Italy

${ }^{29}$ The University of Mississippi, University, MS 38677, USA

${ }^{30}$ Charles Sturt University, Wagga Wagga, NSW 2678, Australia

${ }^{31}$ Caltech-CaRT, Pasadena, CA 91125, USA

${ }^{32}$ Carleton College, Northfield, MN 55057, USA

${ }^{33}$ The University of Melbourne, Parkville VIC 3010, Australia

${ }^{34}$ Cardiff University, Cardiff, CF24 3AA, United Kingdom

${ }^{35}$ Eötvös University, ELTE 1053 Budapest, Hungary

${ }^{36}$ University of Salerno, 84084 Fisciano (Salerno), Italy

${ }^{37}$ The University of Sheffield, Sheffield S10 2TN, United Kingdom

${ }^{38}$ The Pennsylvania State University, University Park, PA 16802, USA

${ }^{39}$ Inter-University Centre for Astronomy and Astrophysics, Pune - 411007, India

${ }^{40}$ Southern University and A\&M College, Baton Rouge, LA 70813, USA

${ }^{41}$ California Institute of Technology, Pasadena, CA 91125, USA

${ }^{42}$ University of Rochester, Rochester, NY 14627, USA

${ }^{43}$ The University of Texas at Austin, Austin, TX 78712, USA

${ }^{44}$ Australian National University, Canberra, 0200, Australia

${ }^{45}$ Embry-Riddle Aeronautical University, Prescott, AZ 86301, USA

${ }^{46}$ University of Minnesota, Minneapolis, MN 55455, USA

${ }^{47}$ University of Adelaide, Adelaide, SA 5005, Australia

${ }^{48}$ University of Southampton, Southampton, SO17 1BJ, United Kingdom

${ }^{49}$ Northwestern University, Evanston, IL 60208, USA

${ }^{50}$ National Astronomical Observatory of Japan, Tokyo 181-8588, Japan

${ }^{51}$ Institute of Applied Physics, Nizhny Novgorod, 603950, Russia

${ }^{52}$ University of Strathclyde, Glasgow, G1 1XQ, United Kingdom

${ }^{53}$ Loyola University, New Orleans, LA 70118, USA

${ }^{54}$ Hobart and William Smith Colleges, Geneva, NY 14456, USA

${ }^{55}$ Louisiana Tech University, Ruston, LA 71272, USA

${ }^{56}$ Andrews University, Berrien Springs, MI 49104, USA

${ }^{57}$ Universitat de les Illes Balears, E-07122 Palma de Mallorca, Spain 
${ }^{58}$ Sonoma State University, Rohnert Park, CA 94928, USA

${ }^{59}$ Trinity University, San Antonio, TX 78212, USA

${ }^{60}$ Rochester Institute of Technology, Rochester, NY 14623, USA

${ }^{61}$ Southeastern Louisiana University, Hammond, LA 70402, USA 


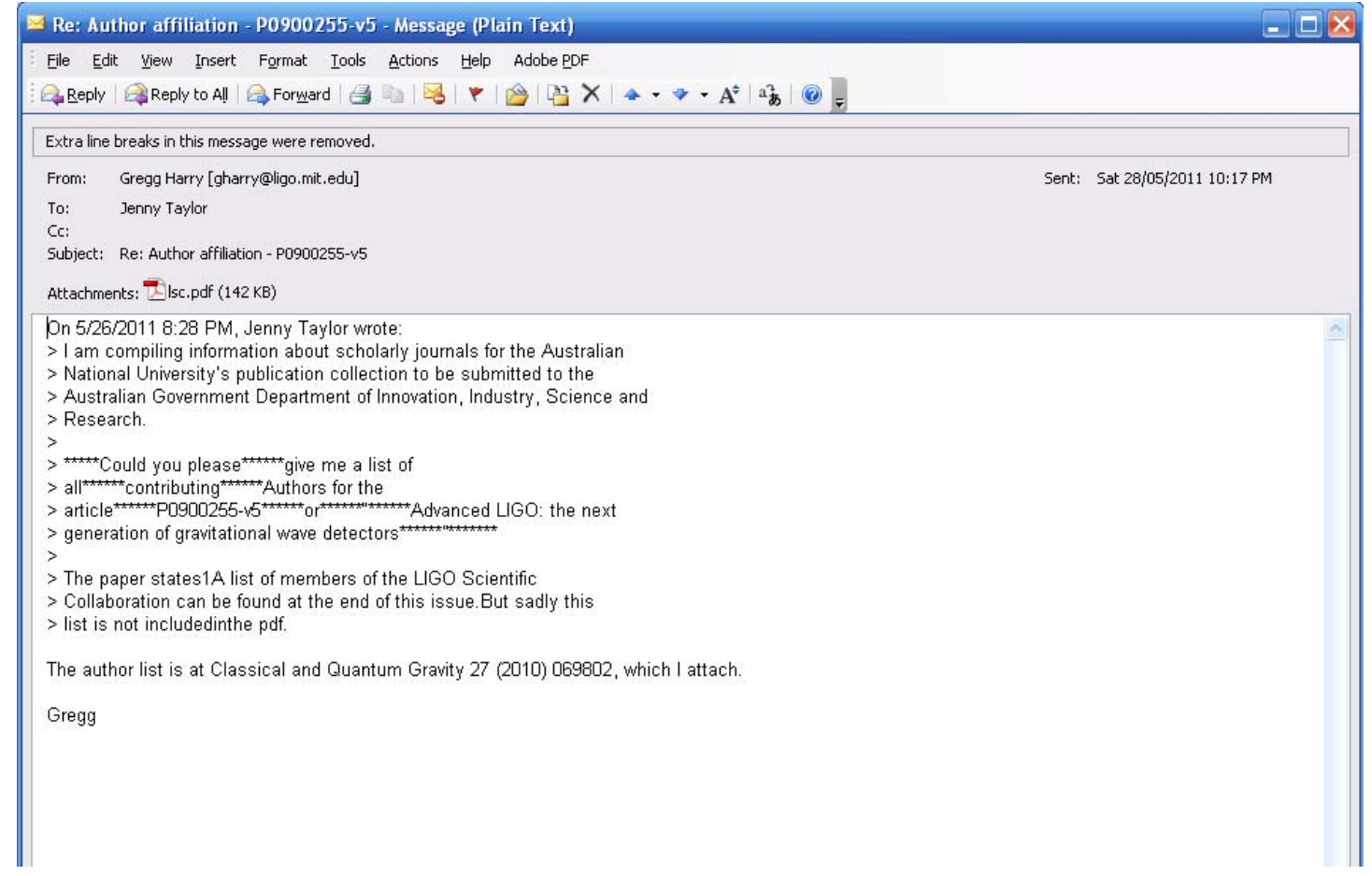

